\title{
Die veränderte Struktur des Arbeitsmarktes: zukunftsfähig oder doch nicht nachhaltig?
}

Deutschland galt noch bis vor wenigen Jahren als der „kranke Mann“ Europas. Die Arbeitslosigkeit erreichte von Rezession zu Rezession neue Höchststände. Seit 2005 zeigt sich ein anderes Bild. Die Arbeitslosigkeit ist seitdem im Sinkflug und selbst die nominell schwerste Wirtschaftskrise in der Nachkriegszeit hinterließ nur wenige Spuren. Deutschland ist in den letzten Jahren mit Blick auf seine Arbeitsmarktperformance zu einem „Vorzeigemodell“ geworden, auch weil die jüngere Entwicklung im internationalen Vergleich ihresgleichen sucht. Der Beitrag sucht nach einer Antwort auf die Frage, was hinter der bemerkenswerten Aufwärtsentwicklung stehen könnte, und erörtert, inwieweit eine nachhaltige Trendwende in Sicht ist. ${ }^{1}$

\section{Einleitung}

Seit 2005 befindet sich der deutsche Arbeitsmarkt in einer verblüffenden Aufwärtsentwicklung. Die Erwerbstätigkeit legte seitdem kräftig zu und wird aller Voraussicht nach im laufenden Jahr einen neuen Rekordwert von jahresdurchschnittlich 41,07 Mio. erreichen (Fuchs et al. 2011). 2005 betrug die Arbeitslosigkeit im Jahresdurchschnitt noch 4,86 Mio. Für 2011 wird allgemein erwartet, dass die Arbeitslosigkeit im Mittel unter 3 Mio. liegen wird. Dazu kommt, dass selbst die schwere Wirtschafts- und Finanzkrise in den Jahren 2008 und 2009 dem positiven Trend so gut wie keinen Abbruch tat. Anders als in früheren Rezessionen erreichte die Arbeitslosigkeit keinen neuen Höchststand und der positive Trend am Arbeitsmarkt vor der Krise setzte sich nach der Krise unvermindert fort.

Angesichts der Positiventwicklung am deutschen Arbeitsmarkt stellen sich mehrere Fragen.

(1) Welche Faktoren stecken dahinter? Mögliche Kandidaten sind eine bessere wirtschaftliche Entwicklung, erste Auswirkungen des demografischen Wandels auf das Arbeitskräfteangebot oder auch institutionelle Veränderungen, wie die Mitte der letzten Dekade vollzogenen Arbeitsmarktreformen, oder auch neue Elemente eines Krisenmanagements.
(2) Handelt es sich bei den Verbesserungen am Arbeitsmarkt eher um eine temporäre Aufwärtsentwicklung oder werden sich diese im Sinne einer Trendwende als nachhaltig erweisen?

(3) Wurden die positiven Veränderungen am Arbeitsmarkt mit nicht intendierten Wirkungen erkauft, die eine längerfristige Stabilisierung des positiven Trends gefährden?

Der vorliegende Beitrag sucht nach Antworten auf die genannten Fragen, indem er zunächst die Arbeitsmarktentwicklung in den letzten Jahren näher beleuchtet (Abschnitt 2). Dabei geht es um den $\mathrm{Zu}$ sammenhang von Wirtschaftswachstum und wichtigen Arbeitsmarktgrößen und um die Identifikation veränderter Reaktionsmuster in der jüngeren Vergangenheit. Im Anschluss daran soll auf Faktoren eingegangen werden, die zur veränderten, positiveren Struktur des deutschen Arbeitsmarktes beigetragen haben könnten (Abschnitt 3). Im Einzelnen geht es dabei um Effekte der Arbeitsmarktreformen, die jüngere Lohnentwicklung und das Krisenmanagement im Rahmen der Wirtschaftsund Finanzkrise. Auf der Basis dessen werden dann im Fazit vorliegende Hinweise zur Nachhaltigkeit der Positiventwicklung und zu ihren möglichen Nebenwirkungen gewürdigt (Abschnitt 4).

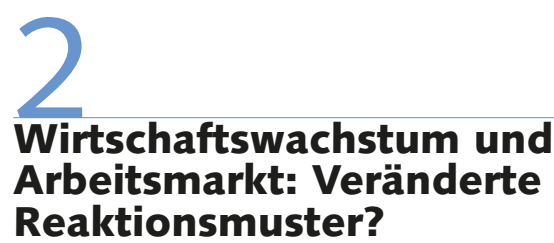

Nachdem die Arbeitslosigkeit seit der Wiedervereinigung zunächst kontinuierlich gestiegen ist, hat sich die Arbeitsmarktlage hierzulande seit 2005 deutlich verbessert. Bezieht man die vorliegenden Prognosen für das laufende Jahr mit ein, könnte die Arbeitslosigkeit von 2005 bis 2011 jahresdurchschnittlich um 1,87 Mio. oder um 4,6 Prozentpunkte sinken (Abbildung 1). Aus heutiger Sicht könnte sich im Jahr 2012 trotz der absehbaren Eintrübung der wirtschaftlichen Entwicklung der positi-

\footnotetext{
1 Der Beitrag ist die überarbeitete und aktualisierte Fassung eines Vortrages, den der Autor auf der Jahrestagung der Deutschen Vereinigung für Sozialwissenschaftliche Arbeitsmarktforschung (SAMF) am 18./19.11.2010 in Cottbus gehalten hat. Der Verfasser bedankt sich bei der anonymen Gutachterin bzw. dem anonymen Gutachter der WSI-Mitteilungen für wertvolle Hinweise zur Überarbeitung des Manuskripts und bei Laura Dittrich für die technische Assistenz.
}

Ulrich Walwei, Dr., ist Vizedirektor des Instituts für Arbeitsmarkt- und Berufsforschung (IAB) Nürnberg. Arbeitsschwerpunkte: Längerfristige Arbeitsmarkttrends, Politikanalysen, Flexibilisierung des Arbeitsmarktes, international vergleichende Institutionenökonomik. e-mail: Ulrich.Walwei@iab.de 
ve Trend am Arbeitsmarkt fortsetzen (vgl. Fuchs et al. 2011; Projektgruppe Gemeinschaftsdiagnose 2011).

Hinweise auf ein verändertes Reaktionsmuster des Arbeitsmarktes seit Mitte der letzten Dekade liefert auch eine genauere Betrachtung des Trends und der zyklischen Komponente der Arbeitslosigkeitsentwicklung (Abbildung 2). Dabei zeigt sich zweierlei: Der Trend ist seit 2005 rückläufig. Zudem ist in der Aufschwungphase 2005 bis 2008 der stärkste konjunkturbedingte Rückgang der Arbeitslosigkeit seit 1991 und in der nachfolgenden Abschwungphase ein - verglichen mit früheren Rezessionen - relativ schwacher zyklischer Anstieg zu beobachten. Vieles spricht für das Erreichen einer Trendwende am Arbeitsmarkt, denn trotz der schweren Wirtschafts- und Finanzkrise erreichte die Arbeitslosigkeit nicht wieder einen neuerlichen Höchststand.

Die bemerkenswerte Verbesserung am deutschen Arbeitsmarkt zeigt sich auch im internationalen Vergleich. Während Deutschland 2005 noch eine sehr hohe Arbeitslosigkeit aufwies, hat sich die Lage bis zum aktuellen Rand auf markante Weise geändert (Abbildung 3). Deutschland ist das westliche Land, in dem in der jüngeren Vergangenheit die Arbeitslosigkeit am stärksten zurückgegangen ist. Auch die auf Erhebungsdaten basierenden Erwerbslosenquoten des internationalen Vergleichs bestätigen damit den insgesamt positiven Trend.

Somit stellt sich die Frage, was hinter der Besserungstendenz am Arbeitsmarkt steht. Eine naheliegende Erklärung könnten bessere volkswirtschaftliche Rahmenbedingungen sein. So würde ein höheres Wirtschaftswachstum - unter sonst gleichen Bedingungen - die Arbeitsnachfrage beflügeln und könnte damit einen Beitrag zum Abbau der Arbeitslosigkeit leisten. Hierfür gibt es aber empirisch nicht wirklich überzeugende Belege. Das reale Wirtschaftswachstum ist von 1992 bis 2010 jahresdurchschnittlich um etwas mehr als $1,3 \%$ gewachsen. Betrachtet man die beiden Zeiträume 1992 bis 2000 und 2001 bis 2010, so fiel das jahresdurchschnittliche Wirtschaftswachstum im ersten Zeitraum mit fast 1,8\% beinahe doppelt so hoch aus wie im zweiten Zeitraum (0,9\%). Und auch die Periode 2005 bis 2010 lag mit einem jahresdurchschnittlichen Wirtschaftswachstum von gut $1,1 \%$ nicht wesentlich darüber. ${ }^{2}$

\footnotetext{
2 Die angegebenen Durchschnittswerte basieren auf Daten des Statistischen Bundesamts.
}

\section{Abb. 1: Anzahl Arbeitslose 2000-2011}

- in 1.000 und in \% der Erwerbspersonen -

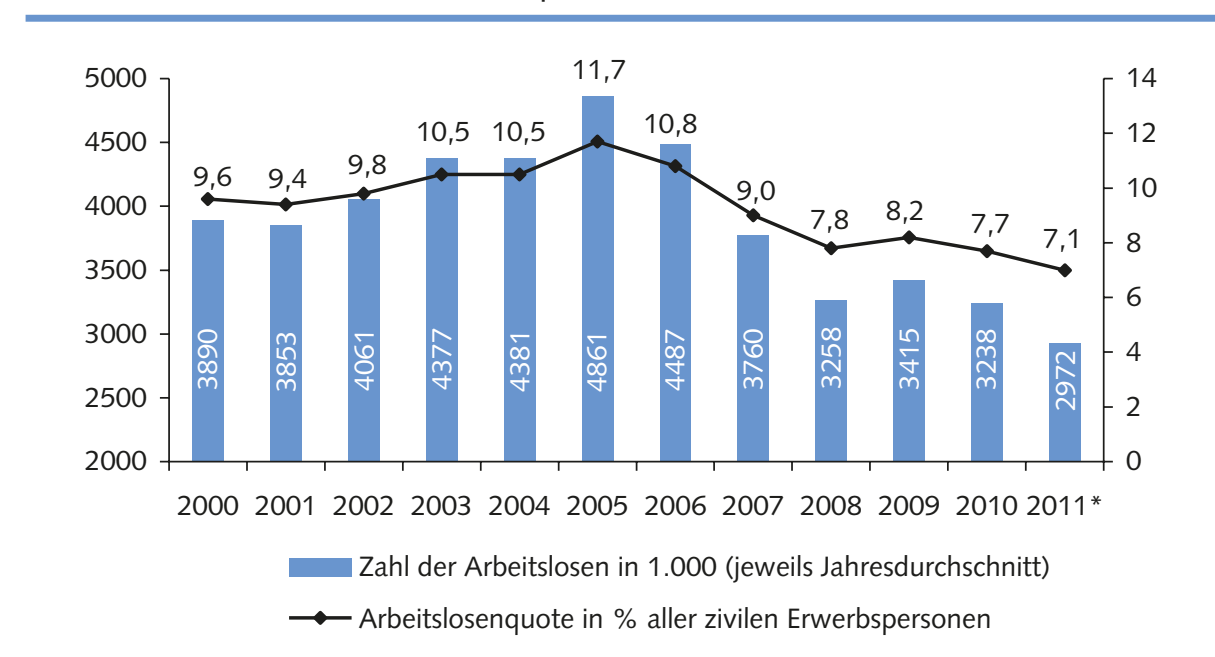

* IAB-Prognose.

Quelle: Bundesagentur für Arbeit; Berechnungen des IAB

WSI MITTEILUNGEN

Abb. 2: Trend und zyklische Komponente der Arbeitslosigkeit 1991-2010 - jeweils in 1.000 -

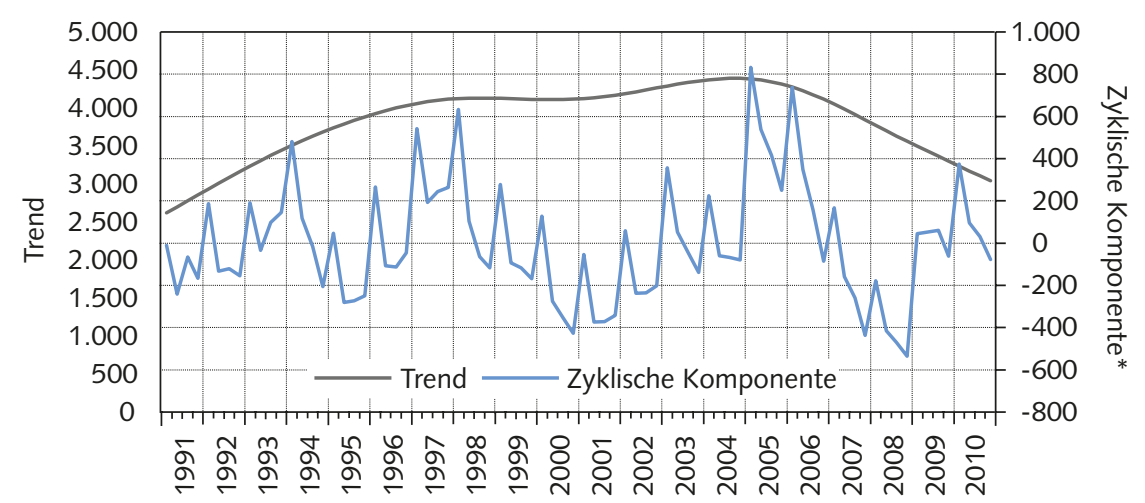

*Zyklische Komponente berechnet mit dem Hodrick-Prescott-Filter (Glättungsparameter $\lambda=1600$ ) auf Basis von Quartalsdaten. Dabei wurden aus den Monatswerten der Arbeitslosigkeit Quartalsdurchschnitte gebildet.

Quelle: Bundesagentur für Arbeit; Berechnungen des IAB.

WSI MITTELUNGEN

Abb. 3: Arbeitslosenquote in ausgewählten Ländern 2005

und 2010 - jeweils in \% der Erwerbspersonen -

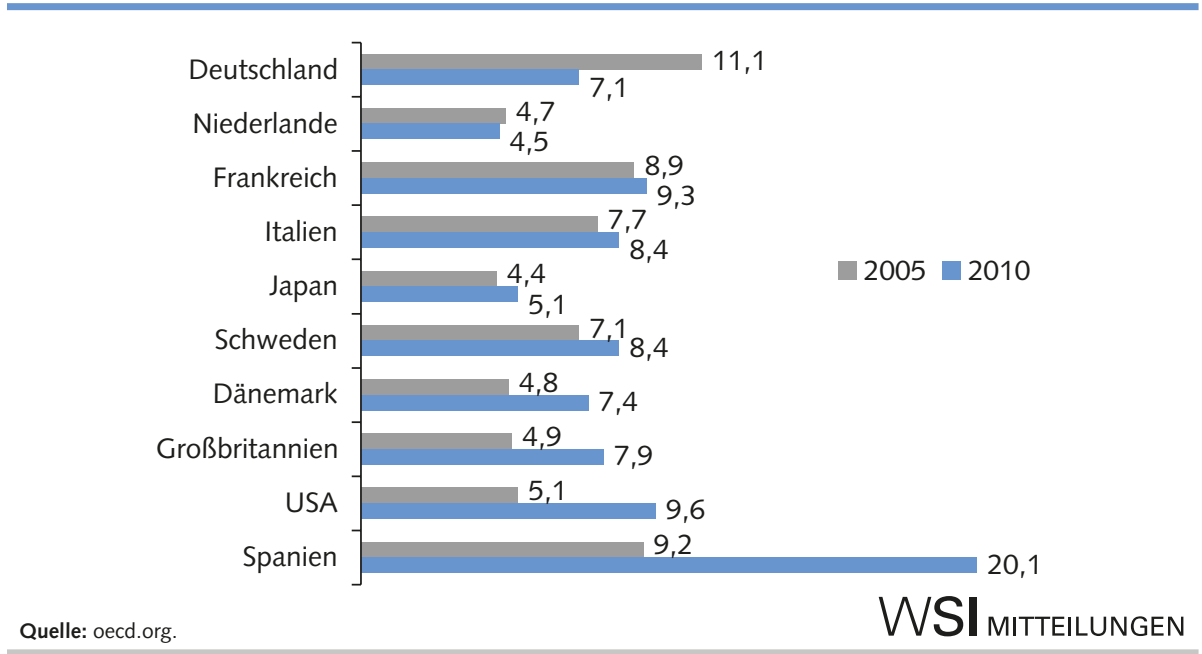


Abb. 4: Trend und zyklische Komponente des Bruttoinlandsprodukts, 1. Quartal 1991 - 1. Quartal 2011 (Index $2000=100$ )

- Wachstumsraten in \% im Vergleich zum Vorquartal, saisonbereinigt -

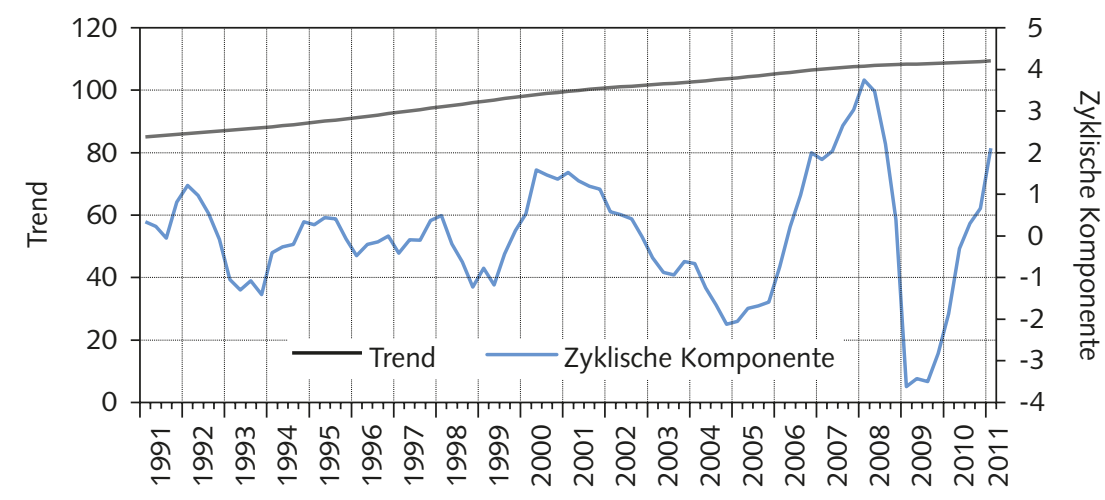

*Zyklische Komponente berechnet mit dem Hodrick-Prescott-Filter (Glättungsparameter $\lambda=1600$ ) auf Basis von Quartalsdaten Dabei wurden aus den Monatswerten der Arbeitslosigkeit Quartalsdurchschnitte gebildet.
Abb. 5. Entwicklung des Erwerbspersonenpotenzials 1991-2011 - in $1.000-$

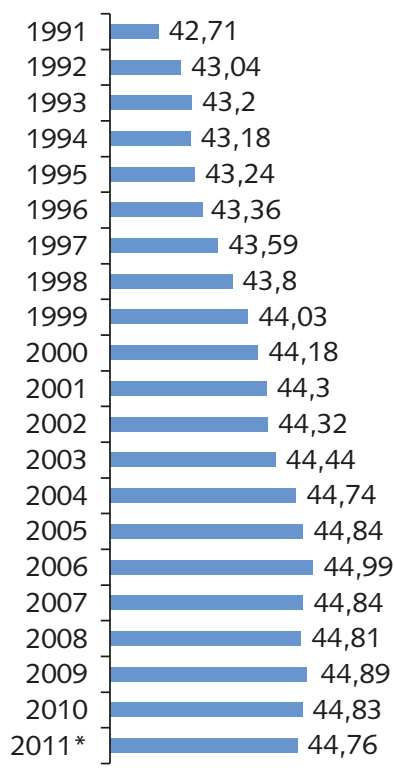

*Prognose für 2011: mittlere Variante des IAB.

Quelle: Fuchs et al. 2011 und IAB-A2.

\section{WSI MITTEILUNGEN}

Auch die Zerlegung der Entwicklung des Bruttoinlandsprodukts (BIP) in Trend und zyklische Komponente weist nicht auf einen besonderen Wachstumsschub hin (Abbildung 4). Zwar ist der Aufschwung 2005 bis 2008 durch eine kräftige zyklische Komponente gekennzeichnet, die aber durch den dann folgenden schweren Einbruch infolge der Wirtschafts- und Finanzkrise in großen Teilen wieder zunichte gemacht wurde.
Eine zweite mögliche Erklärung für die bessere Arbeitsmarktentwicklung in den letzten Jahren könnte auf der Seite des Arbeitskräfteangebots liegen. Wenn man am Arbeitsmarkt nicht von perfekten Bedingungen und damit einer hohen Anpassungsfähigkeit ausgeht, macht es zumindest in der kurzen Frist einen Unterschied, ob mehr oder weniger Arbeitskräfte zur Verfügung stehen. Berechnungen des Instituts für Arbeitsmarkt- und Berufsforschung (IAB) zum Erwerbspersonenpotenzial belegen markante Veränderungen in den letzten Jahren. Das gesamtdeutsche Erwerbspersonenpotenzial ist seit 1991 zunächst kontinuierlich gestiegen und erreichte 2006 mit 44,99 Mio. einen vorläufigen Höchststand (Abbildung 5). Danach zeigt sich eine leicht rückläufige Entwicklung des Erwerbspersonenpotenzials, das 2011 bei 44,76 Mio. liegen dürfte. Selbst wenn Interdependenzen zwischen Arbeitskräfteangebot und Arbeitskräftenachfrage vernachlässigt werden, könnte der Potenzialrückgang angesichts seiner Größenordnung bestenfalls einen kleinen Beitrag zur Verbesserung der Arbeitsmarktlage geleistet haben.

Das Erwerbspersonenpotenzial wird sich den vorliegenden Projektionen zufolge zukünftig weiter verringern und dies mit zunehmender Tendenz (Fuchs/ Dörfler 2005). Weniger Arbeitskräfte bedeuten aber nicht zwangsläufig, dass die Arbeitslosigkeit in gleichem Umfang abnehmen muss. Denn eine rückläufige Bevölkerung und ein zurückgehendes Arbeitskräfteangebot können sich negativ auf den Kapitalstock auswirken sowie Investitionen und Konsum bremsen (Ottaviano/ Perri 2006; Brücker/Jahn 2010). Die Arbeitskräftenachfrage dürfte auch deshalb in Mitleidenschaft gezogen werden, weil Engpässe in bestimmten Segmenten des Arbeitsmarktes zu Lohnsteigerungen führen können.

Als dritte Erklärung für die zuletzt günstigere Beschäftigungssituation kommt eine veränderte Erfassung der Arbeitslosigkeit in Betracht. So könnte es sein, dass durch arbeitsmarktpolitische Maßnahmen und eine stärkere Aktivierung von Arbeitslosen vermehrt Personen aus der registrierten, offenen Arbeitslosigkeit in die verdeckte Arbeitslosigkeit (Stille Reserve) übergegangen sind. Jedoch gibt es auch hierfür keinen Beleg. Während die Zahl der registrierten Arbeitslosen von 2005 bis 2010 von jahresdurchschnittlich 4,86 Mio. auf 3,24 Mio. zurückgegangen ist, verharrte die Stille Reserve im selben Zeitraum bei gut 1,4 Mio (Fuchs et al. 2011). Somit geht die Abnahme der Unterbeschäftigung (Summe aus registrierten Arbeitslosen und Stiller Reserve) allein auf eine niedrigere Arbeitslosigkeit zurück.

Schließlich sind noch Veränderungen der Arbeitsnachfrage zu würdigen. Da nicht zuletzt aufgrund der schweren Wirtschafts- und Finanzkrise das Wirtschaftswachstum als entscheidender Impuls für eine stärkere Arbeitsnachfrage weitgehend auszuschließen ist, könnte sich aber unabhängig davon der Beschäftigungstrend verändert haben. Das Wirtschaftswachstum wäre somit beschäftigungsintensiver geworden. Hierfür finden sich in der Tat einige Hinweise. So legten zwischen 2005 und 2010 - jeweils im Jahresdurchschnitt - die Erwerbstätigkeit um knapp 1,65 Mio. bzw. 4,2\%, die sozialversicherungspflichtige Beschäftigung um fast 1,52 Mio. bzw. 5,8\% und das Arbeitsvolumen um 1,74 Mrd. Stunden bzw. 3,1\% zu. Abbildung 6 illustriert die Entwicklung der drei Größen seit 1991 und dokumentiert den insbesondere zuletzt starken Aufwärtstrend von sozialversicherungspflichtiger Beschäftigung und Arbeitsvolumen. Besonders bemerkenswert ist dabei, dass sich die sozialversicherungspflichtige Beschäftigung nach einer Phase des starken Rückgangs der Erwerbsform Anfang der letzten Dekade wieder erholt hat und damit maßgeblich zum Abbau der Arbeitslosigkeit beigetragen haben dürfte. Dabei stellt sich aber die Frage, was wiederum diese neuerliche Ent- 
wicklung getrieben haben könnte. Hierauf soll im nachfolgenden Abschnitt eingegangen werden.

\section{Verbesserter Beschäfti- gungstrend: Mögliche Erklärungsansätze}

Die deutlich verbesserte Arbeitsmarktlage seit 2005 ist - wie im vorhergehenden Abschnitt ausgeführt-in erster Linie dem starken Wachstum der sozialversicherungspflichtigen Beschäftigung zu verdanken. Dies ist bemerkenswert, weil seitdem nicht nur eine Phase des wirtschaftlichen Aufschwungs bis zum 1. Quartal 2008, sondern auch eine schwere Wirtschaftsund Finanzkrise mit nachfolgender Erholung ins Land gegangen ist. Von außerordentlich günstigen wirtschaftlichen Rahmenbedingungen in den letzten Jahren kann also keine Rede sein. Von daher ist nach weiteren Faktoren zu suchen, die den Beschäftigungstrend positiv beeinflusst haben könnten. Dabei ist die Identifikation möglicher Einflussfaktoren eine große wissenschaftliche Herausforderung. Anders als auf der mikroökonomischen Ebene, wo mit Kontrollgruppen gearbeitet werden kann, gibt es auf der gesamtwirtschaftlichen Ebene keine vergleichbaren Ansätze zur Ermittlung kausaler Effekte. In Ermangelung von Modellen, die zweifelsfrei gesamtwirtschaftliche Zusammenhänge identifizieren können, kann es hier also allenfalls darum gehen, Indizien für die Relevanz bestimmter Einflussfaktoren zu benennen. Im Einzelnen geht es im Folgenden um die möglichen Wirkungen der Arbeitsmarktreformen, veränderter lohnpolitischer Rahmenbedingungen und des jüngsten Krisenmanagements.

\subsection{ARBEITSMARKTREFORMEN}

In der zweiten Legislaturperiode der rotgrünen Bundesregierung von 2002 bis 2005 wurden umfassende Arbeitsmarktreformen verabschiedet, die in Teilen noch heute kontrovers diskutiert werden. Sie umfassen die sogenannten Hartz-Reformen und weitere Maßnahmen im Rahmen der Agenda 2010. Wichtige Elemente waren Veränderungen der Arbeitsmarktpolitik im engeren Sinne, Anpassungen der Leistungen bei Arbeitslosigkeit (wie dem Ar- beitslosengeld sowie der Arbeitslosen- und Sozialhilfe), Erleichterungen bei flexiblen Beschäftigungsformen (wie z.B. Mini-Jobs, Leiharbeit und befristeter Beschäftigung) sowie eine Deregulierung des Kündigungsschutzes bei Kleinbetrieben.

Für einen möglichen Effekt der Arbeitsmarktreformen spricht zunächst die Entwicklung der Arbeitslosenquote seit 2005, die einen Rückgang der strukturellen Arbeitslosigkeit nahelegt. Erstmals seit der Wiedervereinigung in Gesamtdeutschland und seit den frühen 1970er Jahren in Westdeutschland ist die Arbeitslosigkeit nicht mehr von Abschwung zu Abschwung gestiegen. Der Automatismus von konjunkturellem Abschwung und Anstieg der Sockelarbeitslosigkeit wurde zumindest vorerst unterbrochen. Der Sachverständi- genrat zur Begutachtung der gesamtwirtschaftlichen Entwicklung kam in seiner letzten Schätzung der inflationsstabilen Arbeitslosigkeit, der sog. NAIRU, auf einen Rückgang um etwa zwei Prozentpunkte (Sachverständigenrat 2008).

Auch die Entwicklung der BeveridgeKurve legt einen Rückgang der strukturellen Arbeitslosigkeit nahe. Sie bildet den Zusammenhang zwischen Vakanzen und Arbeitslosen ab. Strukturelle Änderungen führen mittelfristig zu einer günstigeren Relation von offenen Stellen zu Arbeitslosen und damit zu einer Verschiebung der Kurve nach links oben. Genau eine solche Verschiebung zeigt sich am aktuellen Rand, die selbst durch die schwere Wirtschafts- und Finanzkrise nicht konterkariert wurde (Abbildung 7). Der Shift der

\section{Abb. 6: Entwicklung von Arbeitsvolumen, Erwerbstätigkeit und sozialversicherungspflichtiger Beschäftigung 1992-2010 \\ - jeweils Veränderung gegenüber Vorjahr in \% -}

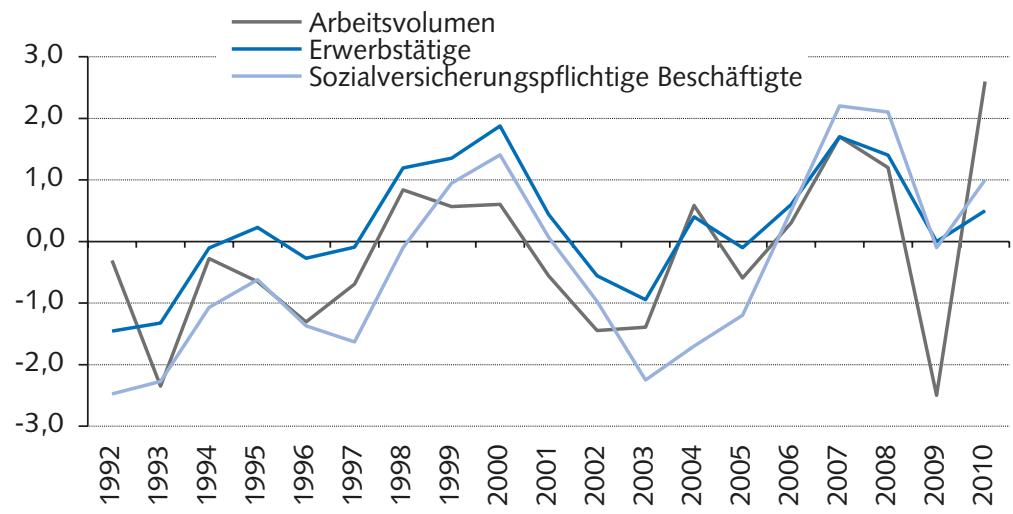

Quelle: Berechnungen des IAB-A2

WSI MITTELUNGEN

\section{Abb. 7: Beveridge-Kurve}

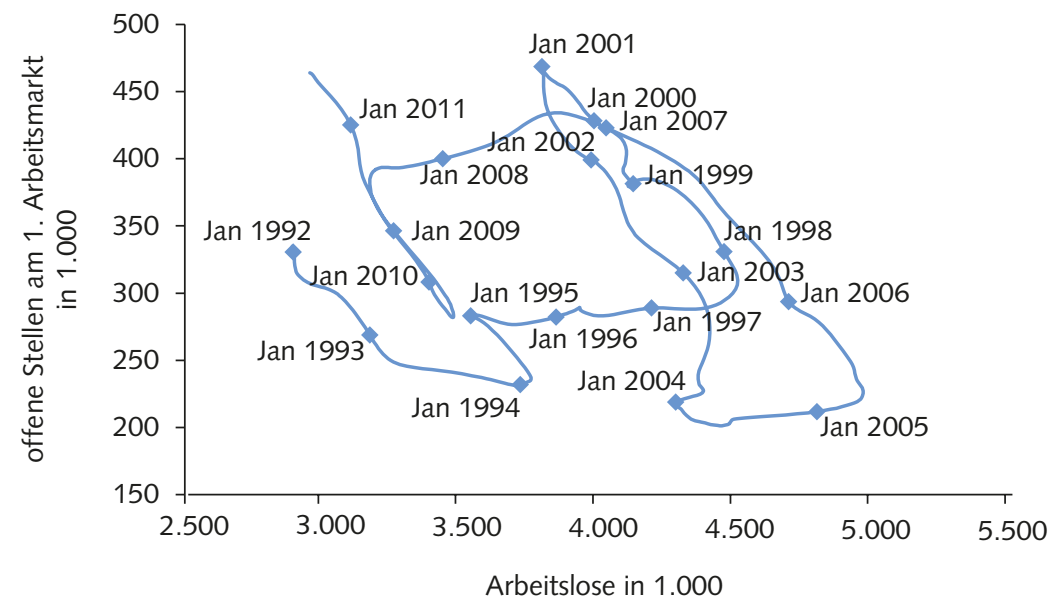

Quelle: BA-Statistik.

WSI MITTELUNGEN 


\section{Abb. 8: Entwicklung von Bruttoinlandsprodukt (BIP) und}

Labour-Turnover-Rate ${ }^{1}$ 1996-2010
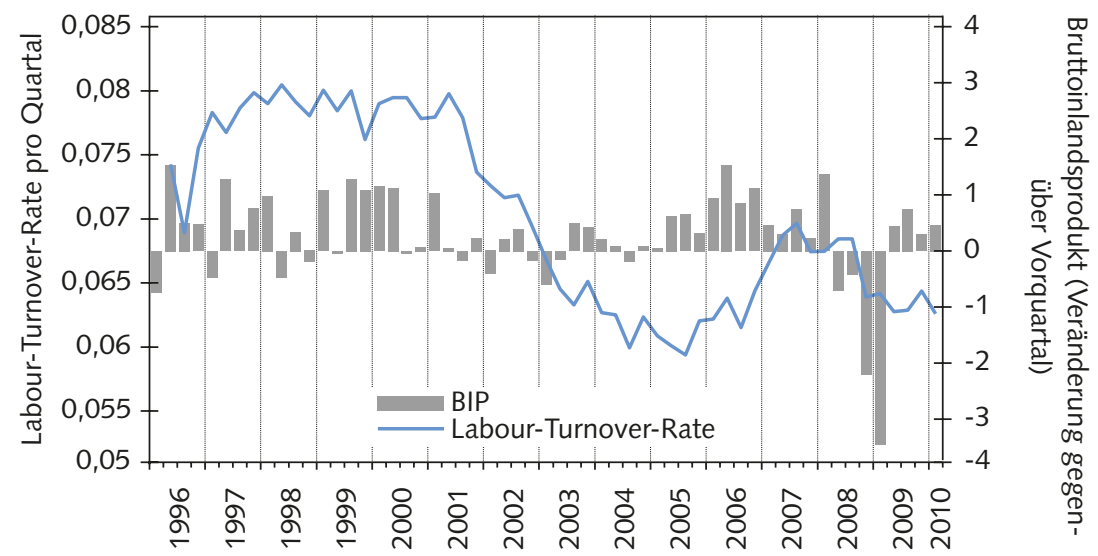

1 Labour-Turnover-Rate bezeichnet den Anteil der Summe aus begonnenen und beendeten Beschäftigungsverhältnissen an dem Bestand aller Beschäftigungsverhältnisse.

Quelle: IAB, FB-A1, Rothe

WSI MITTELUUNGEN

Abb. 9: Verteilungsspielraum und seine Ausschöpfung - in \% -

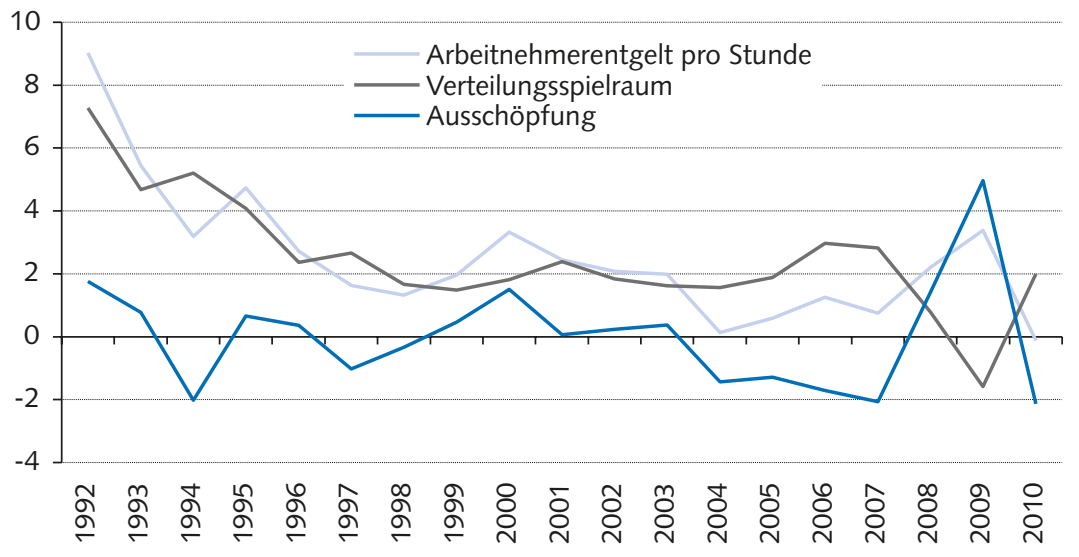

Anmerkungen: Berechnung des Verteilungsspielraums nach dem Konzept des Sachverständigenrats zur Begutachtung der gesamtwirtschaftlichen Entwicklung.

Beveridge-Kurve ist als Hinweis für eine verbesserte Matchingeffizienz zu deuten, d.h. Unternehmen und Arbeitsuchende finden offenbar besser zusammen.

Für verbesserte Beschäftigungschancen Arbeitsloser in Folge der Arbeitsmarktreformen gibt es mehrere mögliche Gründe. Sie reichen von einer höheren Effektivität arbeitsmarktpolitischer Maßnahmen und ihrer Administration, über einen erleichterten Arbeitsmarktzugang durch flexible Beschäftigungsformen, bis hin zu einer verstärkten Aktivierung und Eigenverantwortung von Arbeitslosen. Auf der Makroebene lassen sich diese Teilaspekte freilich nicht trennen. Ein wesentliches Moment könnte aber die allgemein gestiegene Suchintensität von Stellenbewerbern sein. Ergebnisse aus der Erhebung des gesamtwirtschaftlichen Stellenangebots deuten darauf hin, dass die Bereitschaft von Bewerbern gestiegen ist, auch Stellen unterhalb ihres jeweiligen Qualifikationsniveaus zu niedrigeren Löhnen oder schlechteren Arbeitsbedingungen (z.B. Arbeitszeiten, Anfahrt) anzunehmen. Insbesondere Betriebe, die solche Konzessionen der Bewerber berichtet hatten, konnten auch ihre Stellen leichter besetzen (Kettner/Rebien 2007; vgl. dazu auch Rebien/Kennter 2011). Dabei ist zu bedenken, dass Veränderungen bei den Geldleistungen (wie z.B. durch Hartz IV) nicht nur die Konzessionsbereitschaft der unmittelbar betroffenen Arbeitslosen be- einflussen, sondern auch die Zugeständnisse von Personen, die sich durch (Langzeit-) Arbeitslosigkeit bedroht sehen.

Die Zunahme atypischer Erwerbsformen und die wachsende Lohnungleichheit sind Begleiterscheinungen der Arbeitsmarktreformen. ${ }^{3}$ Die Effekte und Ursachen sind aber alles andere als klar. Zunächst gibt es keinen Beleg, dass durch die größere Verbreitung atypischer Erwerbsformen die Instabilität der Beschäftigung insgesamt zugenommen hätte. Die Zahl der begonnenen und beendeten Beschäftigungsverhältnisse war im letzten Aufschwung sogar niedriger als in früheren Boomphasen (Abbildung 8). Dies schließt natürlich nicht aus, dass einzelne Arbeitnehmergruppen zuletzt von stärkerer Instabilität betroffen waren. $\mathrm{Ob}$ dies vornehmlich aus der Art ihres Beschäftigungsverhältnisses resultiert oder aber in erster Linie einer individuell geringen Beschäftigungsfähigkeit geschuldet ist, bedarf der weiteren Forschung (vgl. z. B. Dütsch 2011). Genauso wenig können die Veränderungen der Beschäftigungsqualität unzweifelhaft mit den Arbeitsmarktreformen in Verbindung gebracht werden. Denn der Trend zu einer gewissen Dualisierung und stärkeren Ausdifferenzierung des deutschen Arbeitsmarktes hatte bereits in den 1990er Jahren begonnen (Eichhorst et al. 2009; Dietz/Walwei 2010; Dustmann et al. 2009).

\subsection{LOHNPOLITISCHE RAHMEN- BEDINGUNGEN}

Ein weiterer wichtiger Faktor für die Beschäftigungsentwicklung ist die von den Arbeitsmarktreformen nicht völlig unabhängige Lohnentwicklung. Sie kann zum Aufbau von Arbeitsplätzen beitragen, wenn sie den Verteilungsspielraum nicht ausschöpft und sich damit der Anstieg der Lohnstückkosten in Grenzen hält. Der Verteilungsspielraum ist allgemein definiert als die Summe aus trendmäßigem Wachstum der Arbeitsproduktivität und der Inflationsrate. Abbildung 9 illustriert die Ausschöpfung des Verteilungsspielraums von 1992 bis 2010. Dabei wird deutlich, dass Phasen, in denen der Verteilungsspielraum nicht voll ausgeschöpft wird, nicht etwas vollkommen Neues sind. Deshalb könnte argumentiert werden, dass angesichts der ungünstigen Arbeitsmarktentwicklung bis 2003 die Bedeutung einer

\footnotetext{
3 Zur Lohnungleichheit finden sich in Abschnitt 3.2 nähere Ausführungen.
} 
moderaten Lohnpolitik für die Beschäftigung nicht überschätzt werden dürfe. Neu ist aber, und dies ist für die Einschätzung der aktuellen Entwicklung wichtig, dass insbesondere in der Phase von 2003 bis 2007 - und damit selbst in einer Periode mit kräftigem Aufschwung - an der Lohnzurückhaltung festgehalten wurde. In der Boomperiode von 1998 bis 2001 zeigte sich dagegen eine solche Konstellation noch nicht. Gerade die moderate Lohnpolitik in Zeiten des Booms dürfte die Aufnahmefähigkeit des Arbeitsmarktes in den letzten Jahren erhöht haben.

Ein ähnliches Bild ergibt sich, wenn die Entwicklung der realen Lohnstückkosten hierzulande mit der in anderen Ländern, hier dem Durchschnitt der Europäischen Union (EU) und der Organisation für wirtschaftliche Entwicklung und $\mathrm{Zu}$ sammenarbeit (OECD), verglichen wird (Abbildung 10). Während die realen Lohnstückkosten im Mittel der entwickelten Volkswirtschaften in der letzten Dekade kontinuierlich gestiegen sind, gingen diese in Deutschland seit 2003 zurück und blieben dann bis zum Beginn der Wirtschafts- und Finanzkrise und damit in Zeiten des wirtschaftlichen Aufschwungs auf niedrigem Niveau. Danach führte der krisenbedingte Produktivitätsrückgang zu einem sprunghaften Anstieg der Lohnstückkosten, auch in Deutschland.

Für die in den letzten Jahren ausgesprochen moderate Lohnentwicklung gibt es mehrere Gründe. Wichtige Faktoren sind dabei der sinkende gewerkschaftliche Organisationsgrad, die abnehmende Tarifbindung und tarifliche Öffnungsklauseln. Aber auch die Arbeitsmarktreformen dürften die lohnpolitischen Rahmenbe- dingungen beeinflusst haben und zwar in mehrfacher Hinsicht. Erwähnt wurde bereits die wachsende Konzessionsbereitschaft der Bewerber, die auch in der Akzeptanz niedrig entlohnter Beschäftigung zum Ausdruck kommt. Darüber hinaus haben die Arbeitsmarktreformen die Nutzung spezifischer Beschäftigungsformen erleichtert, die wie Mini-Jobs oder Zeitarbeit mit einer relativ geringen Stundenentlohnung einhergehen (Kalina/Weinkopf 2010; Jahn 2011). Schließlich dürfte die in Folge der Arbeitsmarktreformen geringere Absicherung bei Arbeitslosigkeit die Verhandlungsposition der Arbeitnehmer und ihrer Gewerkschaften verschlechtert haben.

\subsection{KRISENMANAGEMENT}

Als ein weiteres stabilisierendes Element für die Beschäftigung in den letzten Jahren muss das Krisenmanagement im
Zusammenhang mit der schweren Wirtschafts- und Finanzkrise genannt werden. Angesichts eines historischen Rückgangs des realen Bruttoinlandsprodukts 2009 von rund 5\% erwies sich der Arbeitsmarkt als außerordentlich robust. Die Arbeitslosigkeit stieg nur geringfügig und die Erwerbstätigkeit blieb nahezu stabil. Zurückzuführen ist dies auf Maßnahmen der betriebsinternen Arbeitszeitflexibilität und eine temporär geringere Arbeitsproduktivität, die in der Summe als Arbeitskräftehorten gedeutet werden können. Dies soll im Folgenden durch empirische Ergebnisse illustriert werden.

Trotz gewisser Einschränkungen können Veränderungen in der Arbeitsproduktivität pro Kopfbzw. pro Stunde als Indikator für eine mehr oder weniger starke Auslastung des Faktors Arbeit interpretiert werden (vgl. Dietz et al. 2011). Die Abbildungen 11a und $11 b$ zeigen die sich nach einer Trendbe-

\section{Abb. 10: Entwicklung der realen Lohnstückkosten 1. Quartal 2000 - 4. Quartal 2010 (Index 2005 = 100)}

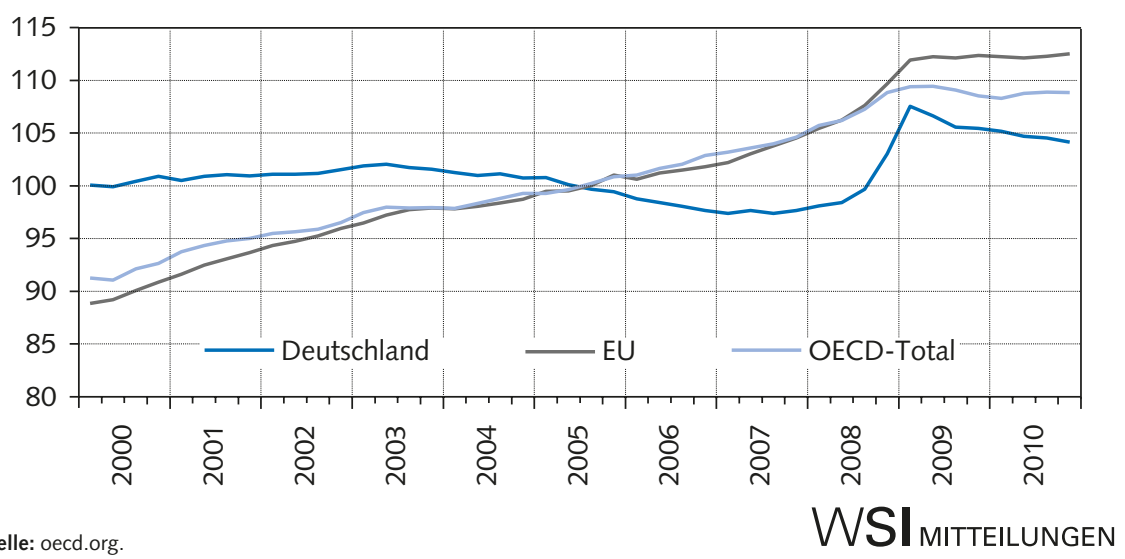

\section{Abb. 11: Zyklische Komponente der Arbeitsproduktivität pro Stunde und pro Kopf}

1. Quartal 1991 - 4. Quartal 2010 - Wachstumsraten im Vergleich zum Vorquartal, saisonbereinigt -

a) Arbeitsproduktivität pro Stunde

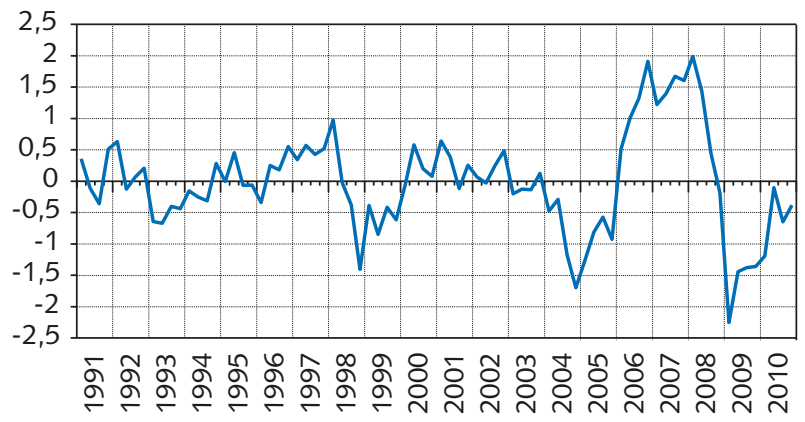

b) Arbeitsproduktivität pro Kopf

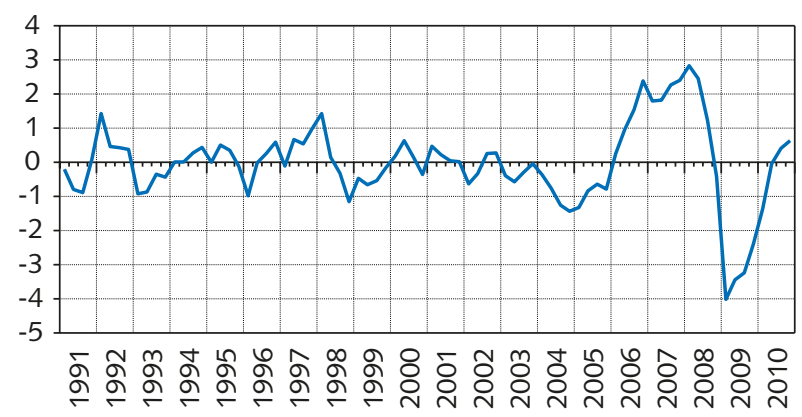

*Zyklische Komponente berechnet mit dem Hodrick-Prescott-Filter (Glättungsparameter $\lambda=1600$ ) auf Basis von Quartalsdaten. 
Abb. 12: Auslastung des Faktors Arbeit in Köpfen und nach Sektoren, 1. Quartal 2005 - 4. Quartal 2010

- Zyklische Komponente der Arbeitsproduktivität pro Erwerbstätigen*

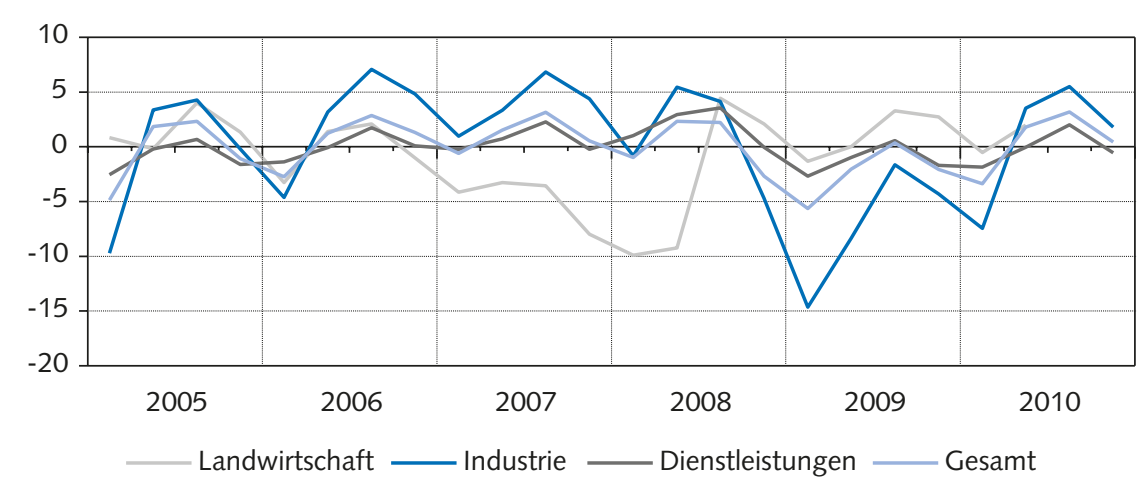

*Zyklische Komponente berechnet mit dem Hodrick-Prescott-Filter (Glättungsparameter $\lambda=1600$ ) auf Basis von Quartalsdaten Quelle: Statistisches Bundesamt; Berechnungen des IAB. WSI MITTELUUNGEN

reinigung ergebende zyklische Komponente der Arbeitsproduktivität pro Kopf und pro Stunde. Dabei signalisieren positive Abweichungen (oberhalb der Null-Linie) einen intensiveren Arbeitseinsatz und negative Abweichungen (unterhalb der Null-Linie) ein Arbeitskräftehorten. Letzteres ließ sich seit der Wiedervereinigung durchaus beobachten, allerdings nie in dem Ausmaß, wie es in der zurückliegenden Krise der Fall war.

Die Arbeitsproduktivität variiert aber nicht nur im Zeitablauf, sondern auch nach Branchen. Abbildung 12 zeigt im 1. Quartal 2009 insbesondere im sekundären Sektor, also im Produzierenden Gewerbe (Industrie), starke negative Abweichungen vom Trend. Für diesen Bereich ergeben sich die stärksten Abweichungen seit 1991, was darauf hindeutet, dass Betriebe in diesem Wirtschaftszweig trotz eines erheblichen Nachfrageeinbruchs in besonders starkem Maße Arbeitskräfte gehalten haben. Sie waren auch deshalb in der Lage dazu, weil der vorhergehende Aufschwung ein gewisses Polster geschaffen hatte, was durch die stark positive (zyklische) Abweichung der Arbeitsproduktivität vom Trend in diesen Jahren zum Ausdruck kommt. Auch die bereits angesprochene moderate Lohnpolitik in Phasen des Aufschwungs hat auf der betrieblichen Seite wenig Kostendruck und damit Personalanpassungsbedarf entstehen lassen. Dennoch bleibt die Frage, warum die Betriebe - angesichts der nicht unbeträchtlichen Unsicherheiten zu Beginn der Wirtschafts- und Finanzkrise weitgehend an ihren Stammbelegschaften festgehalten haben.
Um Arbeitskräftehorten in der Realität zu erklären, muss man sich von den Standardannahmen der ökonomischen Theorie lösen. Denn in einer Welt mit vollkommenen Märkten, homogener Arbeit und ohne Transaktionskosten würde eine Unterauslastung der Produktionsfaktoren unweigerlich zu Freisetzungen führen. Denn andernfalls würden die Betriebe aus dem Markt gedrängt. In der realen Welt immer komplexer werdender Arbeitsbeziehungen und der wachsenden Wissensgesellschaft sind aber Freisetzungen mit (mehr) Kosten verbunden. Sie betreffen die Entlassung, die Motivation der verbleibenden Belegschaft sowie mögliche Wiedereinstellungs- und Wiedereinarbeitungskosten. Die Betriebe standen also zu Beginn der Wirtschafts- und Finanzkrise vor einer Güterabwägung. Die eine Möglichkeit bestand darin, sich von Personal zu trennen und dabei das Risiko der Wiederbeschaffung bei verbesserter Auftragslage in Kauf zu nehmen. Die andere Möglichkeit war diejenige, unterausgelastetes Personal zu halten und dabei das Risiko einer sich nicht wieder erholenden Güternachfrage in Kauf zu nehmen. Die Betriebe entschieden sich in den allermeisten Fällen für die zweite Alternative, weil sie ihre Wettbewerbsfähigkeit und Marktchancen als weiterhin gut einstuften, weil sie auf ein rasches Ende der Krise setzten und weil sie in der vorhergehenden Aufschwungphase bereits beträchtliche Schwierigkeiten bei der Rekrutierung von Fachkräften wahrgenommen hatten und mit ähnlichen Problemen im Falle einer wirtschaftlichen Erholung rech- nen mussten. Vor allem durch betriebsinterne Formen der Flexibilität, wie dem Zurückfahren von Arbeitszeitkonten und Überstunden, der Vereinbarung flexibler Wochenarbeitszeiten sowie insbesondere durch die Inanspruchnahme staatlicher Hilfen wie der Kurzarbeit, konnten in den von der Krise betroffenen Betrieben wertvolle Stammkräfte gehalten werden.

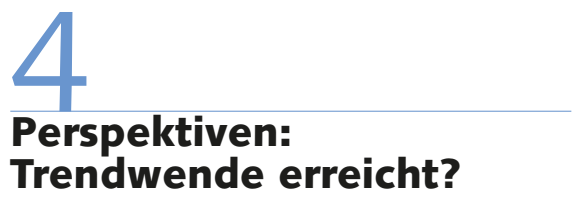

Der deutsche Arbeitsmarkt befindet sich gemessen an wichtigen Indikatoren eindeutig auf dem Weg der Besserung. Die Arbeitslosigkeit geht nicht nur insgesamt zurück, sondern auch ihr Sockel baut sich ab. Dies kommt insbesondere darin zum Ausdruck, dass die Arbeitslosigkeit am Ende des letzten Abschwungs erstmals seit Langem niedriger lag als am Ende des vorhergehenden Abschwungs. Der positive Trend ist im Kern weder auf ein besonders kräftiges Wirtschaftswachstum noch auf geänderte Erfassungsregeln bei der Arbeitslosigkeit oder gar auf den demografischen Wandel zurückzuführen. Entscheidend für die Besserung am Arbeitsmarkt ist die positive Entwicklung der Erwerbstätigkeit und dabei insbesondere der zuletzt wieder stark aufwärtsgerichteten sozialversicherungspflichtigen Beschäftigung.

Der günstigere Beschäftigungstrend ist mit herkömmlichen wissenschaftlichen Methoden nur unzureichend zu erklären, zumal es in den Analysen schwer fällt, einzelne Erklärungsfaktoren zu isolieren. Die vorliegenden Indizien deuten darauf hin, dass der positive Trend der Beschäftigung nicht monokausal bestimmbar ist. Vielmehr gibt es mehrere Faktoren, deren Zusammenspiel dem Arbeitsmarkt zugute kam. Zu nennen sind hier die Arbeitsmarktreformen, die die Aufnahmefähigkeit des Arbeitsmarktes, die Konzessionsbereitschaft der Bewerber und die Suchaktivitäten von Arbeitslosen erhöht haben. Dazu kommt eine beschäftigungsfreundliche Lohnpolitik, die insbesondere in Aufschwungzeiten die Arbeitsnachfrage beflügelt und im Abschwung den Kostendruck gebremst hat. Schließlich hat auch das erfolgreiche Krisenmanagement im Kontext der Wirtschafts- und Finanzkrise einen neuerlichen Anstieg der Sockel- 
arbeitslosigkeit verhindert. Qualifiziertes und gut eingearbeitetes Personal wurde gehalten und man kann in diesem Zusammenhang sogar von einem beschäftigungsorientierten Arbeitskräftehorten sprechen.

Vieles spricht dafür, dass in Deutschland der Mix aus mehr externer Flexibilität (durch die Arbeitsmarktreformen) und mehr intern-numerischer Flexibilität (im Zuge des Krisenmanagements) mit einem verbesserten Arbeitsmarkttrend einhergeht. Während die Arbeitsmarktreformen vornehmlich dem Beschäftigungsaufbau im Dienstleistungsbereich und auch Outsidern zugutekamen, verhinderte das Krisenmanagement einen starken Arbeitsplatzabbau im Verarbeitenden Gewerbe und begünstigte gut eingearbeitete und qualifizierte Stammbelegschaften.

Mit Blick auf die mittelfristigen Perspektiven bedeutet dies alles aber nicht, dass weitere Verbesserungen am Arbeitsmarkt ein Selbstläufer wären. Eine zentrale Voraussetzung ist zunächst, dass die wirtschaftliche Entwicklung hierzulande stabil bleibt. Deutschland ist wie kaum ein anders Land mit der Weltwirtschaft verflochten. Es wird auch zukünftig in hohem Maße von seinen Exporten abhängig sein und ist damit auf den Erhalt seiner Wettbewerbsfähigkeit angewiesen. $\mathrm{Zu}$ bedenken ist zudem, dass inländische Akzente in der Wirtschaftspolitik insbesondere im Euroraum immer ein hohes Gewicht haben werden. Gerade die Wirtschafts- und Finanzkrise hat gezeigt, wie anfällig die deutsche Wirtschaft für Turbulenzen auf weltwirtschaftlicher Ebene ist. Auch die zuletzt um sich greifende Schuldenkrise und die zu ihrer Bewältigung notwendigen Konsolidierungsbemühungen der um ihre Bonität bemühten Euroländer wie auch der Vereinigten Staaten von Amerika kann sich zumindest mittelfristig als Hemmschuh für wirtschaftliche Dynamik entwickeln. Deshalb muss Deutschland ein hohes Interesse daran haben, dass den Ursachen solcher Krisen (wie der unzureichenden Regulierung der Finanzmärkte oder auch den teils überbordenden Staatsschulden) soweit wie möglich der Boden entzogen wird.

Eine andere wichtige Herausforderung in der längeren Frist ist die weiter rückläufige Zahl verfügbarer Arbeitskräfte. Sie nimmt zwar Druck vom Arbeitsmarkt, senkt aber nicht automatisch die Arbeitslosigkeit. Vielmehr ist damit zu rechnen, dass sich die Arbeitsnachfrage Zug um Zug an das Erwerbspersonenpotenzial anpasst, weil insgesamt weniger investiert wird, dadurch der Kapitalstock abnimmt und sich aufgrund von Engpässen bei der Fachkräfterekrutierung durch Lohnreaktionen und mögliche Produktionshemmnisse negative Auswirkungen auf die Arbeitsnachfrage ergeben können.

Ein weiteres Problem sind schließlich die unverkennbaren Dualisierungstendenzen am Arbeitsmarkt und damit die Frage nach seiner Durchlässigkeit. Analysen zu atypischen Beschäftigungsformen und dem Niedriglohnbereich verweisen immer wieder auf ein beträchtliches Niveau an Drehtür- und Einsperreffekten. Für die Nachhaltigkeit des Aufschwungs am Arbeitsmarkt und die Reduzierung des noch immer großen harten Kerns der Arbeitslosigkeit wird es deshalb darauf ankommen, die Beschäftigungsfähigkeit wettbewerbsschwacher Personengruppen zu verbessern. Wichtige Elemente sind dabei nicht nur geeignete Formen einer berufsbegleitenden Qualifizierung, sondern auch Maßnahmen der sozialen Flankierung von Beschäftigung wie gezielte $\mathrm{Ab}$ gabensenkungen im Niedriglohnbereich oder auch moderate Mindestlöhne.

\section{LITERATUR}

Brücker, H./Jahn, E. J. (2010): Arbeitsmarktwirkungen der Migration: Einheimische Arbeitskräfte gewinnen durch Zuwanderung, IAB-Kurzbericht (26), Nürnberg

Dietz, M./Stops, M./Walwei, U. (2011): Safeguarding jobs in times of crisis - lessons from the German experience, International Institute for Labour Studies, Discussion paper (207), Genf

Dietz, M./Walwei, U. (2010): Mehr Beschäftigung um jeden Preis?, in: Kaudelka, K./Kilger, G. (Hrsg.): Die Arbeitswelt von morgen. Wie wollen wir leben und arbeiten?, Bielefeld, S. 57-87

Dütsch, M. (2011): Wie prekär ist Zeitarbeit? Eine Analyse mit dem Matching-Ansatz, in: Zeitschrift für Arbeitsmarktforschung 43 (4),

S. 299-318

Dustmann, Ch./Ludsteck, J./Schönberg, U. (2009): Revisiting the German wage structure, in: The Quarterly Journal of Economics 124 (2), S. 843-881

Eichhorst, W./Marx, P./Thode, E. (2009): Arbeitsmarkt und Beschäftigung in Deutschland 2000-2009, hrsg. von der Bertelsmann Stiftung, Gütersloh

Fuchs, J./Dörfler, K. (2005): Projektion des Erwerbspersonenpotenzials bis 2050 - Annahmen und Datengrundlage, IAB-Forschungsbericht

(25), Nürnberg

Fuchs, J./Hummel, M./Hutter, C./Klinger, S./Spitznagel, E./Wanger,

S./Weber, E./Zika, G. (2011): Prognose 2011/2012: Schwere Zeiten für den Arbeitsmarkt, IAB-Kurzbericht (19), Nürnberg
Projektgruppe Gemeinschaftsdiagnose (Hrsg.) (2011): Gemeinschaftsdiagnose 2011: Aufschwung setzt sich fort - Europäische Schuldenkrise noch ungelöst, http://ftp.zew.de/pub/zew-docs/gutachten/gdfruehjahr2011.pdf

Jahn, E. J. (2011): Entlohnung in der Zeitarbeit, in: IAB-Forum (1), S. 40-49, Nürnberg

Kalina, Th./Weinkopf, C. (2010): Niedriglohnbeschäftigung 2008: Stagnation auf hohem Niveau - Lohnspektrum franzt nach unten aus, IAQ-Report (010-06), Duisburg/Essen

Kettner, A./Rebien, M. (2007): Soziale Arbeitsgelegenheiten - Einsatz und Wirkungsweise aus betrieblicher und arbeitsmarktpolitischer Perspektive, IAB-Forschungsbericht (02), Nürnberg

Ottaviano, G./Peri, G. (2006): The Economic Value of Cultural Diver-

sity: Evidence from U.S. Cities, in: Journal of Economic Geography 6 (1), S. 9-44, NBER Working Paper Version 10904

Rebien, M./Kettner, A. (2011): Die Konzessionsbereitschaft von Bewerbern und Beschäftigten nach den Hartz-Reformen, in: WSI-Mitteilungen 64 (5), S. 218-225

Sachverständigenrat zur Begutachtung der gesamtwirtschaftlichen Entwicklung (2008): Jahresgutachten 2008/09: Die Finanzkrise meistern - Wachstumskräfte stärken, http://www.sachverstaendigenratwirtschaft.de/fileadmin/dateiablage/download/gutachten/ga08_ges. pdf 